\title{
On Compact Steep Spectrum Sources and Jet Production
}

\section{Ezeugo Jeremiah Chukwuemerie}

Department of Physics and Industrial physics, NnamdiAzikiwe University, Awka, Anambra State, Nigeria

Email address:

chuksemeria@yahoo.com

To cite this article:

Ezeugo Jeremiah Chukwuemerie. On Compact Steep Spectrum Sources and Jet Production. American Journal of Astronomy and Astrophysics. Vol. 3, No. 1, 2015, pp. 1-5. doi: 10.11648/j.ajaa.20150301.11

\begin{abstract}
In this work, we show the possibility that jet production by magnetization predominates over other mechanisms of jet production in radio sources with low power output. We obtain estimates of accretion induced magnetic field and jet-driving magnetic field of compact steep spectrum sources in our sample using analytical methods.A possible implication of the results obtained through simple linear regression analyses of the data estimated for the two aforementioned fields is that jet production by magnetization predominates in the CSS radio sources with lower power output; while in those with higher power output, the converse may be the case - some other processes,such as; hydrodynamic, thermal, and radiation pressures may predominate over jet magnetization.
\end{abstract}

Keywords: Jet, Accretion, Magnetization, Steep Spectrum, Radio Sources

\section{Introduction}

Compact steep spectrum (CSS) sourcesare intrinsically small extragalactic radio sources $[1,2,3,4,5,6]$. Theyconsist of radio galaxies and radio loud quasars but on sub-galactic dimensions. Their observed linear sizes, $D$, are given by $D \leq 20 \mathrm{kpc}$ assuming Hubble constant, $H_{0}=$ $75 \mathrm{kms}^{-1} \mathrm{Mpc}^{-1}$, and deceleration parameter, $q_{0}=0.0$. Their radio luminosity, $P$, at $5 \mathrm{GHz}$ is greater than $10^{25.5} \mathrm{WHz}^{-1}[2,3,5,7]$. They have steep high frequency spectrum of spectral index, $a \geq 0.5\left(S_{v} \sim v^{-a}\right.$; where $S_{v}$ is flux density and $v$ is observing frequency). Their spectral turnover is believed to be due to synchrotron self-absorption in a compact component with a large magnetic field. Generally, they have low polarization at both radio and optical bands, usually $<1 \%[2,3,8,9]$.Their proportion is high; from about $15 \%$ to $30 \%$, depending on the selection frequency, among distant $(z>0.2)$ radio sources of high luminosity $[2,3]$.

Generally, a typical extragalactic radio source takes the shape of a central coresurrounded by two-sided jets. At the end of each jet is located a radio-emitting lobe. The jet is thought to be the conduit through which materials fill the lobe.

In absence of any other mechanism of jet production, many authors have considered that much of the energy powering the jets is derived from the source accretion disks $[4,10$,$] . Gravitational field of a supermassive black hole$ believed to reside at the central core of a radio source attracts its ambient gas particles or clouds[11,12,13]. This causes the gas clouds to accelerate toward the black hole from a range of directions and collide with each other as they approach their orbital trajectory, and finally form an accretion disk. This accretion disk is believed to be the source of jet materials $[4,10,14]$, but the process by which this occurs is not yet fully understood.

This work, therefore,aims at finding if there is any relation between magnetic field possibly responsible for jet formation and that induced by accretion process for possible deductions. We have not applied full magnetohydrodynamic equations which may be considered appropriate in describing the phenomena of accretion and jet production $[10,15,16,17]$. However, for the purpose of obtaining a simple relation between the two fields, we have applied only electromagnetism in our derivations.

The sample of CSS sources used in the analyses is obtained from [3]. The sources are 61 in number, and have linear sizes, $D \leq 20 \mathrm{kpc}$.

\section{Magnetic Field due to Accretion}

Magnetic acceleration model appears to be the most convincing model in explaining astrophysical jet formation $[10,15,16,17]$. This implies that bipolar relativistic jets are powered by magnetic fields tightly twisted by differential rotation of accretion disk [10]. Moreover, it has been pointed out that the terminal velocity of a jet is comparable to the rotational velocity of the disk at the foot of the jet [10]; hence, 
suggesting that a relativistic jet is produced at a position very close to the event horizon of a black hole.

It is commonly believed that accretion disk consists of plasma (i.e. positive ions and electrons). This implies that the effective axial magnetic field generated may either cancel out (assuming equal number of accreting opposite charges) or highly reduced when compared to the field produced if the disk were made up of an ensemble of like charges. However, for the purpose of finding the behavior of jet-driven magnetic field in relation to accretion-induced magnetic field, we assume the disk comprises an ensemble of equal positive and negative charges.

Therefore, approximating the ensemble of negative charges present in the disk to a current-carrying thick hollow circular disk as shown in Fig. 1, then by Biot-Savart law, it can be shown that magnetic field, $B_{a c c}$, produces at the centre of the disk by electrons in the accreting plasma can be expressed as

$$
B_{a c c} \approx \int_{R_{a}}^{R_{b}} \int_{0}^{x}\left(\frac{\mu_{0} I}{2 R}\right) d R d x(1)
$$

where the term in bracket represents magnetic field at the centre of a current-carrying narrow circular ring, $R_{a}$ and $R_{b}$ are inner and outer radii (see Fig.1) respectively, $I$ is total electric current due to the motion of the negative charges (or electrons) in the accreting plasma, $R$ is radius of the disk and $x$ is its thickness.

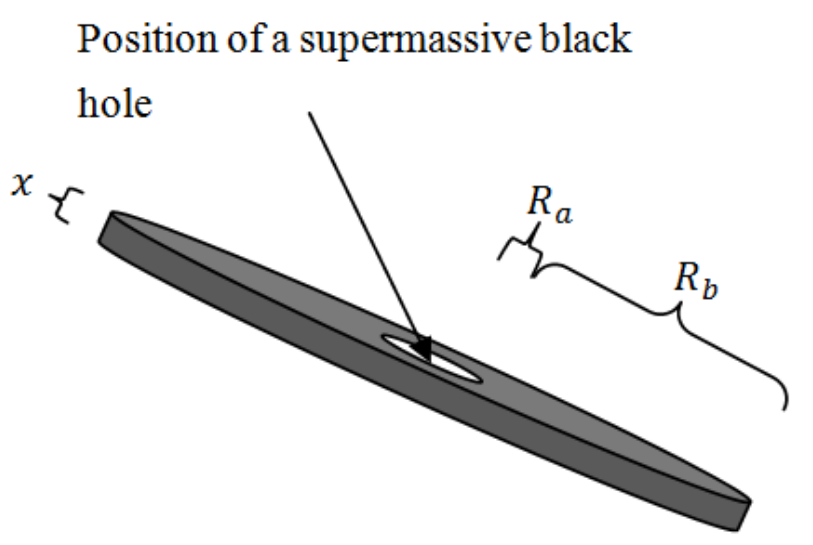

Figure 1. A schematic diagram of assumed uniform accretion disk.

On integration, (1) yields

$$
B_{a c c} \approx \frac{\mu_{0} I x}{2} \ln \left(R_{b}-R_{a}\right)(2)
$$

Applying the general definition of electric current and considering accretion over the source dynamical age, $T,(2)$ may be written as

$$
B_{a c c} \approx \frac{\mu_{0} N e x}{2 T} \ln \left(R_{b}-R_{a}\right)(3)
$$

where $N$ is number of electrons in the disk, and $e$ is electronic charge.

An accretion disk has inner radius whose value may lie between $\approx 1 R_{S}$ and $\approx 10 R_{S}$ depending on the type of disk [14]. $R_{S}$ is Schwarzchild radius or event horizon. The outer radius of accretion disk may extend to $10^{4} R_{s}$ [14]. Taking the mean, $R_{a}$ becomes $5 R_{s}$; while $R_{b}$ gives $10^{4} R_{s}$. Putting the values in (3) yields

$$
B_{a c c} \approx \frac{\mu_{0} N e x}{2 T} \ln \left(9,995 R_{s}\right)(4)
$$

Combining the expression relating Scharzchild radius to black hole mass, $M_{b}$; that is $[14,18]$,

$$
R_{S}=\frac{2 G M_{b}}{c^{2}}(5)
$$

where $G$ is universal gravitational constant, while $c$ is light speed, with (4) and (5), we obtain

$$
B_{a c c} \approx \frac{\mu_{0} N e x}{2 T} \ln \left(19,9950\left[\frac{G M_{b}}{c^{2}}\right]\right)(6)
$$

For simplicity, assuming a source radiating at Eddington limit, the power, $L_{E d d}$, radiated is given by [14]

$$
L_{E d d}=\frac{4 \pi G M_{b} m_{H} c}{\sigma_{T}}(7)
$$

$L_{E d d}$ is in watts, $M_{b}$ is in $\mathrm{kg}, m_{H}$ (proton mass) is in $\mathrm{kg}$ and $\sigma_{T}$ is Thompson interaction cross-section. Approximating Eddington luminosity to source bolometric luminosity, $P_{b o l}$, the last equation becomes

$$
M_{b} \approx \frac{P_{b o l} \sigma_{T}}{4 \pi G m_{H} c}(8)
$$

Putting it in (6), gives

$$
B_{a c c} \approx \frac{\mu_{0} N e x}{2 T} \ln \left(4,997.5\left[\frac{P_{b o l} \sigma_{T}}{\pi m_{H} c^{3}}\right]\right)(9)
$$

Moreover, number of electrons, $N$, in an accretion disk in relation to the particle number density, $n_{a c c}$, of the accreting electrons can be written by

$$
N=n_{a c c} \pi x\left(R_{b}^{2}-R_{a}^{2}\right)(10)
$$

which yields

$$
N \approx 10^{8} \pi x R_{s} n_{a c c}(11)
$$

Combining (5) and (11), we obtain

$$
N \approx 10^{8} \pi x n_{a c c}\left(\frac{2 G M_{b}}{c^{2}}\right)(12)
$$

Also, combining (8) and (12) gives

$$
N \approx\left(5 \times 10^{7}\right) \frac{x n_{a c c} P_{b o l} \sigma_{T}}{c^{3} m_{H}}(13)
$$

Substituting for $N$ in (9) yields

$$
B_{a c c} \approx 2.5 \times 10^{7} \frac{\mu_{0} e x^{2} n_{a c c} P_{b o l} \sigma_{T}}{c^{3} m_{H} T} \ln \left(4,997.5\left[\frac{P_{b o l} \sigma_{T}}{\pi m_{H} c^{3}}\right]\right)(14)
$$

This implies that magnetic field induced at the centre of an accretion disk (for negative charges only) of an extragalactic radio source may be estimated if its thickness $(x)$ and electron number density $\left(n_{a c c}\right)$ are known. However, in terms of field per unit number density, the last equation becomes

$$
\frac{B_{a c c}}{n_{a c c}} \approx 2.5 \times 10^{7} \frac{\mu_{0} e x^{2} P_{b o l} \sigma_{T}}{c^{3} m_{H} T} \ln \left(4,997.5\left[\frac{P_{b o l} \sigma_{T}}{\pi m_{H} c^{3}}\right]\right)(15)
$$


For simplicity, we assume a thin disk with [17]

$$
\frac{\left(\frac{x}{2}\right)}{R_{a}} \approx 0.05(16)
$$

Combining the last equation with (5) and (8), $x$ becomes

$$
x \approx 0.05\left(\frac{P_{b o l} \sigma_{T}}{\pi m_{H} c^{3}}\right)(17)
$$

Substituting for $x$ in (15), we obtain

$$
\frac{B_{a c c}}{n_{a c c}} \approx 6.25 \times 10^{4} \frac{\mu_{0} e \sigma_{T}^{3} P_{b o l}{ }^{3}}{\pi^{2} c^{9} m_{H}^{3} T} \ln \left(4,997.5\left[\frac{P_{b o l} \sigma_{T}}{\pi m_{H} c^{3}}\right]\right)(18)
$$

Moreover, jet-driving magnetic field, $B_{j e t}$, can be expressed as "unpublished" [19]

$$
B_{j e t} \approx \frac{m_{H} c n_{e} \Omega V_{j} T}{\left(1.6 \times 10^{-5}\right) \pi n_{j e t} e D^{2}}(19)
$$

where $n_{e}$ is source's ambient density, $\Omega$ is jet's opening solid angle, $V_{j}$ is jet's velocity, $n_{\text {jet }}$ is jet's internal density, and $D$ is source's observed linear size. In terms of field per unit number density, it becomes

$$
\frac{B_{j e t}}{n_{j e t}} \approx \frac{m_{H} c n_{e} \Omega V_{j} T}{\left(1.6 \times 10^{-5}\right) \pi n_{j e t}^{2} e D^{2}}(20)
$$

\section{Analyses and Results}

In this section, we want to estimate the two quantities, $B_{a c c} / n_{a c c}$ and $B_{j e t} / n_{j e t}$ in (18) and (20) respectively; after which we find if there is any relationship between them using a simple regression analyses. Taking typical values of the following quantities: $m_{H}=1.67 \times 10^{-27} \mathrm{~kg}, e=1.602 \times$ $10^{-19} \mathrm{C}, c=3 \times 10^{8} \mathrm{~m} / \mathrm{s}, \Omega=3.6 \times 10^{-5} \mathrm{sr}, V_{j} \approx 0.3 c[10]$, and $m_{e}=9.109 \times 10^{-31} \mathrm{~kg}, \mu_{0}=4 \pi \times 10^{-7} \mathrm{Hm}^{-1}$, and $\sigma_{T}=2.5 \times 10^{-29} \mathrm{~m}^{2}$, we obtain the following equations;

$$
\frac{B_{a c c}}{n_{a c c}} \approx\left(6.925 \times 10^{-104}\right) \frac{P_{b o l}^{3}}{T} \ln \left(8.82 \times 10^{-25} P_{b o l}\right)(21)
$$

and

$$
\frac{B_{j e t}}{n_{j e t}} \approx\left(2.0158 \times 10^{8}\right) \frac{n_{e} T}{n_{j}^{2} D^{2}}(22)
$$

Using the values of source's observed linear size $(D)$, bolometric luminosity $\left(P_{b o l}[=P v\right.$, where $P$ and $v$ are respectively observed source's luminosity and observing frequency]), source's ages ( $T$, estimated by simply getting the quotient of the individual observed source's linear size with lobe's velocity [0.25c ] of the CSS source, 0710+439, observed by [20]), estimated ambient density $\left(n_{e}\right.$, obtained from [21]) and density of magnetized jet (obtained from "unpublished" [19]), we estimate the two quantities, $B_{a c c} / n_{a c c}$ and $B_{j e t} / n_{j e t}$.

We show $B_{a c c} / n_{a c c}-B_{j e t} / n_{\text {jet }}$ data in Fig. 2. A simple linear regression analysis of the data gives

$$
\log \left(B_{a c c} / n_{\text {acc }}\right)=(20.72)-(1.32) \log \left(B_{\text {jet }} / n_{\text {jet }}\right)(23)
$$

with correlation coefficient, $r \approx 0.54$. Moreover, we carried out a linear regression analysis of $B_{\text {jet }} / n_{\text {jet }}-P$ data (Fig. 3). This yields

$$
\log \left(B_{\text {jet }} / n_{\text {jet }}\right)=(122.8)-(3.64) \log P(24)
$$

with $r \approx 0.44$.

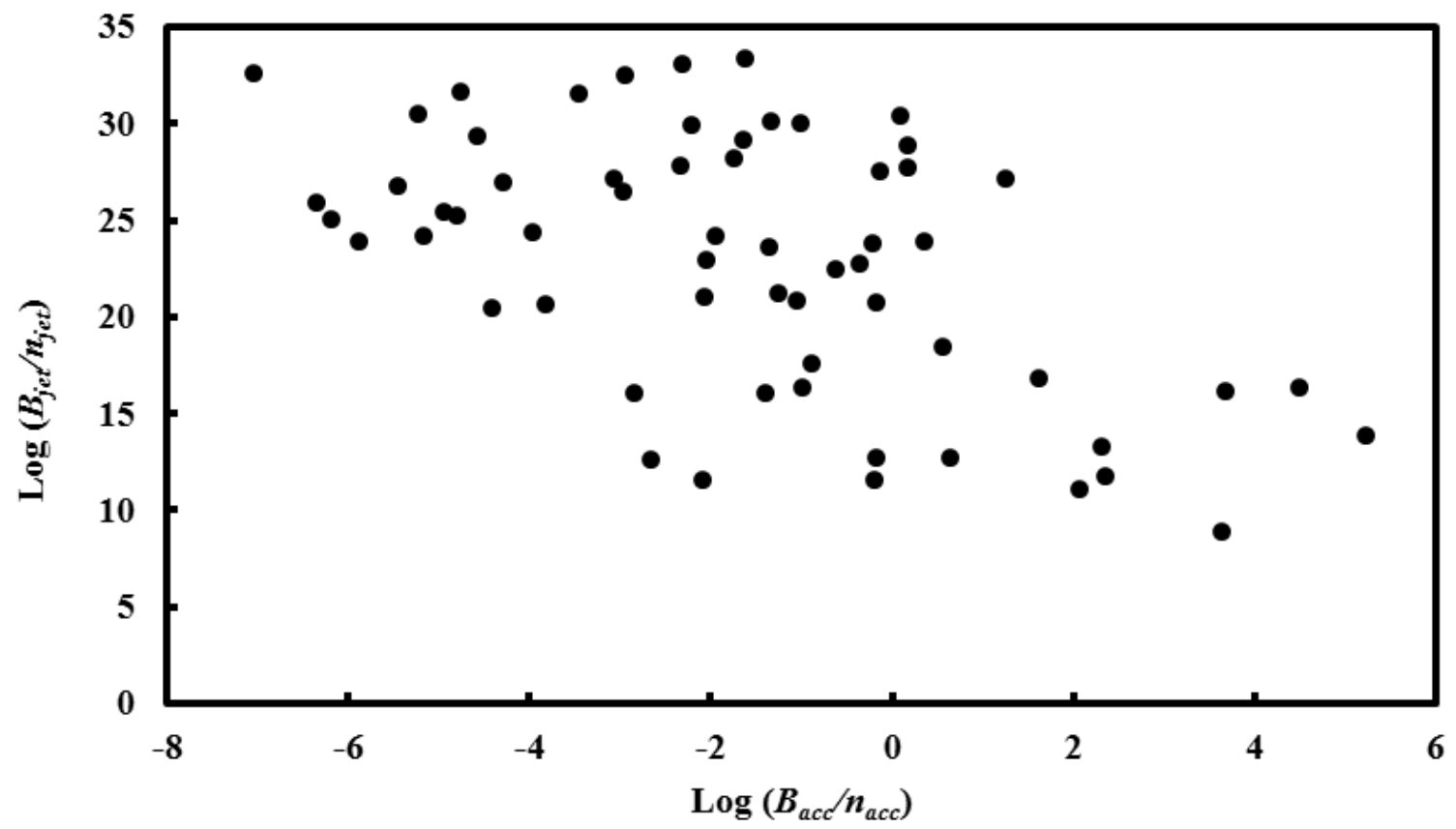

Figure 2. The scatter plot of jet-driving magnetic field per unit density against accretion induced magnetic field per unit density. 


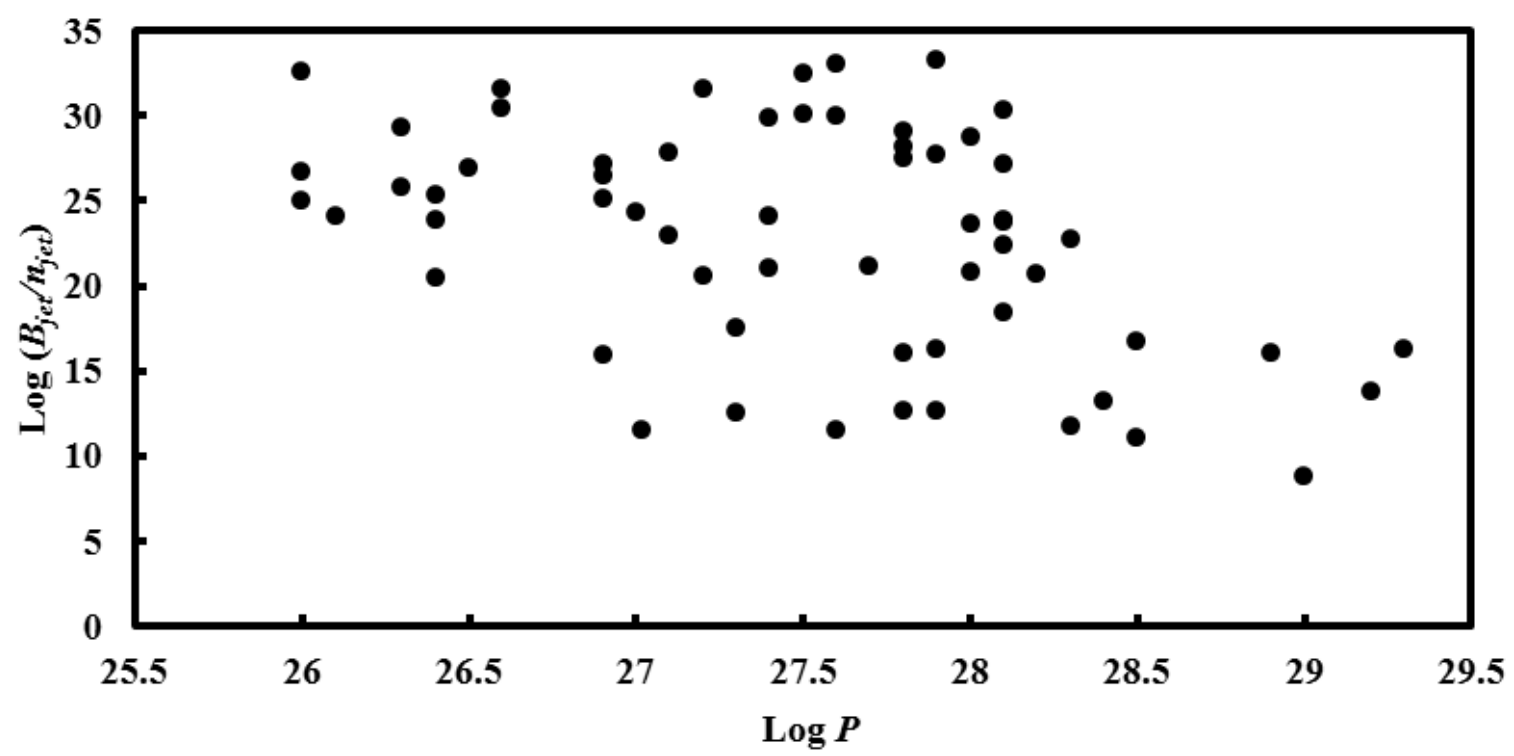

Figure 3. The scatter plot of jet-driving magnetic field per unit density against luminosity.

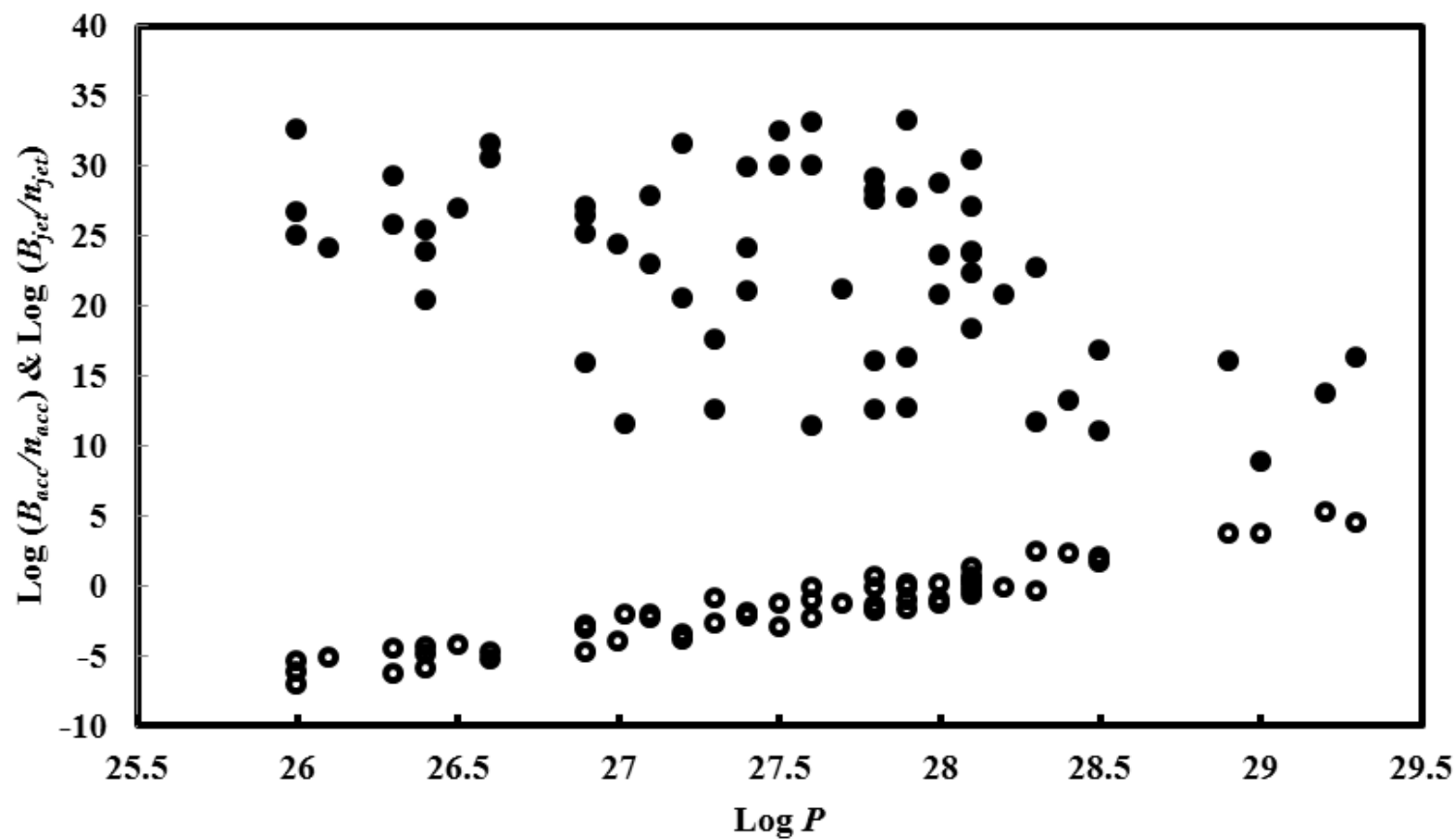

Figure 4. The scatter plot of the accretion induced magnetic field per unit density (open circles) and jet-driving magnetic field per unit density (filled circles) on the same axis.

\section{Discussion and Conclusion}

In the previous sections, we estimate accretion inducedand jet-driving magnetic fields.Using the estimates, we find, through simple linear regression analyses of $B_{j e t} / n_{\text {jet }}-$ $B_{a c c} / n_{\text {acc }}$ data (Fig. 2), a relation between the two fields given by $\left(B_{j e t} / n_{\text {jet }}\right) \sim\left(B_{a c c} / n_{\text {acc }}\right)^{-1.3}$ (where $B_{j e t} \& B_{a c c}$ are respectively jet-driving $\&$ accretion induced magnetic fields; while $n_{j e t} \& n_{a c c}$ are respectively jet's internal \& accretion disk's internal densities), with correlation coefficient, $r=0.54$. This suggestively implies that jet production is predominated by magnetization in the sources with lower power output.In addition, we find through similar regression analyses of a $B_{j e t} / n_{\text {jet }}-P$ data (Fig. 3), a relation of the form, $\left(B_{\text {jet }} / n_{\text {jet }}\right) \sim P^{-3.6}$ (with $r \approx 0.44$ ), between the field and source's luminosity $(P)$.

A possible implication of these results is that jet production by magnetization predominates in the CSS radio sources with lower power output; while in those sources with higher power output, the converse may be the case - some other processes, such as; hydrodynamic, thermal, and radiation pressures may predominate over jet magnetization.

Fig. 4 is the plot of $B_{a c c} / n_{a c c}$ and $B_{j e t} / n_{j e t}$ against radio source's luminosity, $P$, on the same axes. The plot compares $B_{a c c} / n_{a c c}$ and $B_{\text {jet }} / n_{\text {jet }}$ as $P$ increases. It shows that as the source's luminosity grows, accretion 
induced magnetic field increases too, while jet-driving magnetic field decreases.

\section{References}

[1] C. E. Akujor, and S. T. Garrington, "Compactsteep-spectrum sources - polarization observations at1.6, 8.4 and 15GHz," Astron and Astrophy. Suppl. Ser.,vol. 122, pp. 235-255, 1995

[2] C. Fanti, R. Fanti, D. C. Dallacasa, R. T. Schilizzi, R. E.Spencer, and C. Stanghellini, "Are compactsteep-spectrum sources young?”Astron. and Astrophy., vol. 302, pp. 317-326, 1995

[3] C. P. O'Dea, "The compact steep-spectrum sources andGigahertz peaked-spectrum radio sources", Pub. Astron.Soc. Ppacific,vol. 110, pp. 493-532, 1998

[4] C. A. Jackson, "Radio source evolution and unifiedschemes," Publ. Astron. Soc. Aust., vol. 16, pp.124-129, 1999

[5] J. Machalski, M. Jamrozy, and D. J. Saikia, “A multifrequency study of giant radio sources III. Dynamicalage vs. spectral age of the lobes of selected sources",Mont. Not. Roy. Ast. Soc., vol. 395, pp. 812-824, 2009

[6] D. J. Saikia, S. Jeyakumar, P. J. Wiita, H. S. Sanghera,and R. E.Spencer, "Compact steep-spectrum radiosources and unification schemes," Mon. Not. Roy. Ast.Soc., vol. 276, pp. 1215-1223, 1995

[7] C. J. Stanghellini, D. Dallacasa, C. P. O'Dea, S. A.Baum, R. Fanti, C. Fanti, "VLBA observations ofGHz-peaked-spectrum radio sources at 15GHz," Astron. and Astrophy., vol. 377, pp. 377-388, 2001

[8] J. A. Peacock, andJ. V. Wall, "Bright extragalacticradio sources at 2.7GHz-II," Mont. Not. Roy. Ast. Soc.,vol. 198, pp. $843-860,1982$

[9] M. Murgia, R. Fanti, L. Gregorini, U. Klein, K. H.Mark, and M. Vigotti, "Synchrotron spectra and ages ofcompact steep spectrum radio sources," Astron. andAstrophy., vol. 345, pp. 769-777, 1999

[10] S. Koide, K. Shibata, and T. Kudoh, "Relativistic jetformation from black hole magnetized accretion disks,", The Astrophy. Journ., vol. 522, pp.727-752, 1999
[11] M. Mościbrodzka, H. Falcke, H. Shiokawa, and C. F. Gammie, "Observational appearance of inefficient accretion flows and jets in 3D GRMHD simulations: Application to Sagittarius A,"Astron. and Astrophy. vol. 570, A7, 2014

[12] D. Pérez, G. E. Romero, and S. E. P. Bergliaffa, "Accretion disks around black holes in modified strong gravity,"Astron. and Astrophy. vol. 551, A4, 2013

[13] A. Bongiorno, A. Merloni, M. Brusa, B. Magnelli, M. Salvato, M. Mignoli, G. Zamorani, F. Fiore, D. Rosario, V. Mainieri, H. Hao, A. Comastri, C. Vignali, I. Balestra, S. Bardelli, S. Berta, F. Civano, P. Kampczyk, E. Le Floc'h, E. Lusso, D. Lutz, L. Pozzetti, F. Pozzi, L. Riguccini, F. Shankar, andJ. Silverman, "Accreting supermassive black holes in the COSMOS field and the connection to their host galaxies," Mon. Not. Roy Ast. Soc., vol. 427 , pp. 3103-3133, 2012

[14] I. Robson, Active Galactic Nuclei, England: John Wileyand Sons, 1996, pp. 269-307

[15] R. D. Blandford, and D. G. Payne, "Hydromagneticflows from accretion discs and the production of radiojets," Mont. Not. Roy. Ast. Soc., vol. 199, pp. 883-903,1982

[16] G. V. Ustyugova, A. V. Koldoba, M. M. Romanova, V.M. Chechetkin, and R. V. E Lovelace, "Magnetohydrodynamic simulations of outflows from accretiondisks," Astrophys. Journal, vol. 439, pp. L39-L42, 1995

[17] J. E. Pringle, "Accretion disks in astrophysics," Ann.Rev. Astron. astrophy., vol. 19, pp. 137-162, 1981

[18] B. Paczynsky, P. J. Wiita, "Thick accretion disks andsupercritical luminosity," Astro. Astrophy., vol. 88, pp.23-31, 1980

[19] J. C. Ezeugo,"Compact steep-spectrum radio sources and ambient medium density" unpublished.

[20] I. Owsianik, and J. E. Conway, "First detection ofhotspot advance in a compact symmetric object" Astron. and Astrophy., vol. 337, pp. 69-79, 1998

[21] J. C. Ezeugo, and A. A. Ubachukwu, "The spectralturnover-linear size relation and the dynamical evolution of compact steep spectrum sources", Mon. Not.Roy Ast. Soc, vol. 408, pp.2256-2260,2010 